\title{
The value of quantitative diffusion tensor imaging indices of spinal cord disorders
}

\author{
Mohammad Koriem Mahmoud Omar ${ }^{1}$, Abd El-Karem Hasan Abd Allah', Mona Gouda Maghrabi ${ }^{2 *}$ and \\ Mohamed Zidan Mohamed ${ }^{1}$
}

\begin{abstract}
Background: Different lesions affecting the spinal cord can lead to myelopathy. Diffusion tensor imaging (DTI) is widely used to predict the degree of spinal cord microstructure affection and to assess axonal integrity and diffusion directionality. We hypothesized that not all DTI parameters have the same affection with different spinal cord pathologies. The purpose of this study is to assess the value of the quantitative diffusion tensor imaging indices in different spinal cord lesions.

Results: There is highly statistically significant difference of the fractional anisotropy (FA), relative anisotropy (RA), volume ratio (VR) and secondary eigenvector values (E2 and E3) between various studied cord lesions and control levels. There is no statistically significant difference of the apparent diffusion coefficient (ADC) and the primary eigenvector value (E1) (ANOVA test). The ROC curve analysis showed the higher sensitivity and accuracy were ' $88 \%$ and $62.5 \%$, respectively,' with FA cutoff value about 0.380 .
\end{abstract}

Conclusion: The resulted quantitative DTI indices 'fractional anisotropy, relative anisotropy, volume ratio and secondary eigenvalues' work as a numerical in vivo marker of overall tissue injury in different pathologies affecting the spinal cord.

Keywords: MRI, Diffusion tensor imaging, FA, ADC, VR, RA, Eigenvector values, Myelopathy

\section{Background}

Myelopathy defines any neurologic deficit linked to the spinal cord, which usually arises secondary to compression of the spinal cord by osteophyte or herniated disc material, metastatic extradural mass or trauma. Many primary neoplastic tumors and infectious, inflammatory, neurodegenerative, and idiopathic disorders may lead to myelopathy [1].

Magnetic resonance imaging (MRI) is currently the most important modality for imaging of the central nervous system. It offers excellent anatomical information as regards the spinal cord macrostructure [2,3]. MRI has an important role in diagnosing the intramedullary tumors and visualizing its extent and texture [4]. Conventional

\footnotetext{
*Correspondence: kneematony@yahoo.com

${ }^{2}$ Luxor University, Luxor, Egypt

Full list of author information is available at the end of the article
}

MRI provides qualitative information rather than quantitative data $[4,5]$.

The diffusion tensor imaging (DTI) is an advanced noninvasive magnetic resonance imaging technique that allows visualization of the white matter tracts. It allows quantitative evaluation of tissue microstructure integrity by probing the diffusion of water molecules [6-8].

The diffusion tensor imaging (DTI) has quantitative indices, including the apparent diffusion coefficient (ADC) refers to the overall diffusivity of the tissue irrespective of directional dependence, and the fractional anisotropy (FA) measures the fraction of the 'magnitude' of total diffusion that can be assigned to anisotropic diffusion within one particular voxel. It reflects the directional dependence of the diffusion process and is expressed as a relative number ranging from 0 to 1 that increases in relation to the anisotropic diffusion within the tissue being evaluated $[6,9]$. 
Volume ratio (VR) is indicative of the ellipsoid volume in comparison with a sphere with radius equal to the mean diffusivity of the water protons. Consequently, a high VR is indicative of isotropic diffusion [10].

For simplification and practical purposes, the neuron can be expressed as a long ellipsoid, and its vectors are defined as eigenvectors (E1, E2, and E3). The longest vector (E1) along the long axis of the axonal cylinder signifies the longitudinal diffusion in the white matter, while E2 and E3 are minor eigenvectors signifying the transverse diffusion within the white matter of the spinal cord. A high E1 and lesser E2 and E3 values mean highly anisotropic diffusion in the neurons with intact walls [11].

This study hypothesized the specific DTI quantitative parameters to allow in vivo discrimination between various spinal cord lesions with the assessment of the axonal integrity and diffusion directionality.

\section{Methods}

An observational cross-sectional study was done from January 2020 to January 2021. Patients were having various neurological deficits related to the spinal cord and were subjected to detailed clinical history. Their medical reports were revised. All patients underwent conventional spine MRI study as a first investigation tool, and then, diffusion tensor imaging sequence was run for patients with positive conventional MRI findings abnormality affecting the cord.

Inclusion criteria involving patients with abnormal spinal cord findings in conventional MRI.

Exclusion criteria Patients with contraindication for MRI, e.g., an implanted magnetized device, pacemaker and claustrophobia, cerebral palsy and spine radiation and patients with vertebral fixation by screws damaging diffusion sequence by the metal artifacts.

\section{MR imaging technique}

Images were obtained using closed 1.5-Tesla Siemens MRI system (MAGNETOM, Sempra) with 18-channel spine phase array coil. Patients were scanned in the supine position with head first. Patients were asked to reduce movement during the scan.

MRI sequences The following sequence done in the study: sagittal T1WI with (TR/TE: 494/9.2ms, acquisition matrix $320 \times 224$, field of view $=330 \times 100 \mathrm{~mm}^{2}$, number of slices 12 , slice thickness $4 \mathrm{~mm}$, slice gap 20 percent), sagittal T2WI with (TR/TE: 3250/130ms, acquisition matrix $384 \times 269$, field of view $=330 \times 100 \mathrm{~mm}^{2}$, number of slices 12 , slice thickness $4 \mathrm{~mm}$, slice gap 20 percent), axial T2-TSE (TR/TE: 6930/121ms) and sagittal DTI (TR/TE: 5800/105ms) using a single-shot echo-planar imaging sequence, with 20 diffusion with $(b=1000 \mathrm{~s} /$ $\mathrm{mm}^{2}$ ) with additional measurement without diffusion gradient $\left(b=0 \mathrm{~s} / \mathrm{mm}^{2}\right)$, repetition time (TR)/echo time $(\mathrm{TE})=5800 / 105 \mathrm{~ms}$, acquisition matrix $128 \times 128$, field of view $(\mathrm{FOV})=250 \mathrm{x} 100 \mathrm{~mm}^{2}$, number of slices $=36$, slice thickness $2 \mathrm{~mm}$, slice gap $=0$, flip angle $=0$, acquisition time $(\mathrm{TA})=09$ minute 36 seconds. Post-contrast T1-TSE with a dose of $0.1 \mathrm{mmol} / \mathrm{kg}$ of gadolinium that was injected automatically at a rate of $2 \mathrm{ml} / \mathrm{s}$. Contrast was given for patient showing mass like cord lesions, infection or demyelinating disorders.

\section{Imaging processing and analysis}

Diffusion tensor data were post-processed and analyzed on the Syngo MR E11 station (Siemens Healthcare) using the Neuro 3D toolbox by drawing similar region area of interest (ROI) size at area of lesion, areas above and below (controls 1 and 2, respectively); qualitative images including grayscale and color-coded FA, ADC, trace images and $3 \mathrm{D}$ tractogram images were constructed (Figs. 1, 2, 3).

\section{Statistical analysis}

Data were analyzed using Statistical Program for Social Science (SPSS) version $24^{*}$. A $p$ value $>0.05$ was considered statistically significant. Repeated-measure ANOVA test was used to analyze the continuous data and expressed as mean $\pm \mathrm{SD}$. The diagnostic performance of the DTI quantitative parameters at spinal cord lesion versus conventional MRI T2WI-detected signal abnormality was analyzed by ROC curve for calculating cutoff value, sensitivity, specificity, positive, negative values and accuracy.

\section{Results}

Demographic date The study included 31 patients (13 female and 18 male patients) with mean age of $38.23 \pm 19$ years with age range from 15 to 88 years. Twelve patients had spinal cord traumatic lesions, 7 patients with neoplastic spine lesions and 12 patients with demyelinating and infectious cord lesions. Patients were divided into two groups (traumatic and non-traumatic non-degenerative group). The results were compared between all lesion levels and control levels of both groups.

Quantitative results between the normal control levels and the cord lesion level in both groups are given in Tables 1 and 2:

- There was a statistically significant difference between the mean FA value of the traumatic and non-traumatic cord lesion levels and control levels with mean $\pm S D$ values about $(0.420 \pm 0.111$ and $0.471 \pm 0.115$ ), (ANOVA test $p=0.001$ and 0.007 , respectively). 

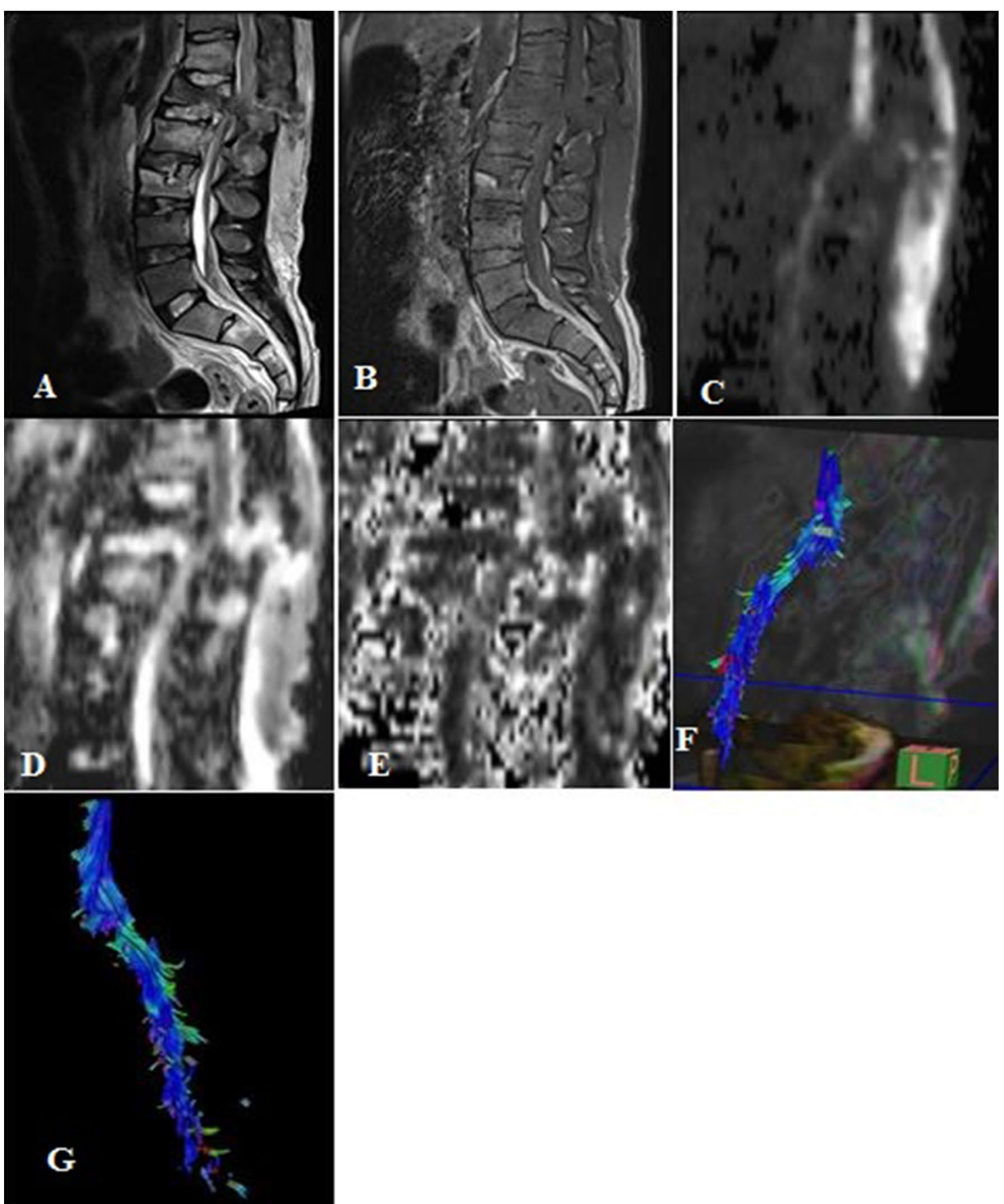

Fig. 1 A 31-year-old male patient, fall from height'palm tree' presented with paraplegia and incontinence. Sagittal T2-weighted (A) and T1-weighted (B) images show compression fracture of $L 1$ vertebral body with posterior retropulsion and posterior paraspinal soft tissue injury including flava, interspinous and supraspinatus ligaments. Large amount of cord edema with high T2 signal intensity and no appreciated T1 signal abnormality. Sagittal trace (C), ADC (D) and grayscale FA (E) images show high trace signal intensity with corresponding low ADC value and low FA signal intensities within the compressed part of the spinal cord. 3D MR fiber tractography images $(\mathbf{F}, \mathbf{G})$ show partial fiber disruption on the posterior aspect of the contused spinal cord 
- There was no statistically significant difference between mean ADC value of the traumatic and non-traumatic cord lesion levels and control levels with mean $\pm S D$ value about $(1.176 \pm 0.245$ and $1.161 \pm 0.121$ ) (ANOVA test $p=0.318$ and 0.220 , respectively).

- There was a statistically significant difference between mean RA value for the traumatic and nontraumatic lesions levels and control levels showed highly significant $R A$ value with mean $\pm S D$ of $(0.375 \pm 0.108$ and $0.434 \pm 0.127)$ (ANOVA test $p=0.001$ and 0.012 , respectively).

- There was a statistically significant difference in the mean VR value between the traumatic and nontraumatic cord lesions and control levels and showed mean $\pm S D$ of $(0.800 \pm 0.086$ and $0.740 \pm 0.116)$ (ANOVA test $p=0.001$ and 0.034 , respectively).

- There was no statistically significant difference between the mean E1 value of the traumatic and non-traumatic cord lesion levels and control levels with mean \pm SD $(1.721 \pm 0.288$ and $1.826 \pm 0.255)$ (ANOVA test $\mathrm{p}=0.444$ and 0.449 , respectively).

- The minor eigenvector values showed statistically significant values with mean \pm SD of E2 were $(1.048 \pm 0.283$ and $0.972 \pm 0.105)$ (ANOVA test $p=0.035$ and 0.006 , respectively). The other minor eigenvector values showed statistically significant values with mean \pm SD of E3 were $(0.778 \pm 0.213$ and $0.700 \pm 0.165 \mathrm{SD}$ ) (ANOVA test $p=0.016$ and 0.047 , respectively).

- When comparing the results between the two study groups of pathology by ANOVA test, the results showed no statistically significant difference in the measured quantitative parameters for FA, ADC, RA with $p$ value $=0.150,0.978$ and 0.167 , respectively. Also, there was no statistical difference between the measured VR, E1, E2 and E3 with $p$ value $=0.213$, $0.416,0.596$ and 0.582 , respectively.

- The ROC curve analysis (Fig. 4 and Table 3 ) showed the FA cutoff value of 0.380 between all lesions and control levels with sensitivity of $88 \%$, specificity of $37 \%$, negative predictive value about $76 \%$, positive predictive value about $58 \%$ and accuracy of $62.5 \%$. The RA cutoff value was 0.370 , which had sensitiv- ity of $77 \%$, specificity of $46 \%$, negative predictive value about $67 \%$, positive predictive value of $59 \%$ and accuracy of $61.5 \%$. The VR cutoff value of 0.640 between all lesions and control levels with sensitivity of $77 \%$, specificity of $52 \%$, negative predictive value of $69 \%$, positive predictive value of $62 \%$ and accuracy of $64.5 \%$.

\section{Discussion}

Diffusion tensor imaging and diffusion tensor tractography have been used successfully to assess the demyelinating or inflammatory disease burden $[12,13]$. DTI can be used to assess the integrity of fiber tract and to guide the management of the spinal cord tumors $[14,15]$. Also, DTI and DTT can be used to assess the functional state and prognosis of traumatic spinal cord lesions [9].

In this study, we found a statistical significant difference between the mean FA, RA, VR, E2 and E3 values between the lesion level and both control levels. We found also no statistical significant difference between the mean of $\mathrm{ADC}$ and $\mathrm{E} 1$ at these levels.

Our result for traumatic cord lesions, the FA and RA showed statistically significant reduction with no statistically significant raised ADC or E1 values. In agree with our result and on studying traumatic spine lesions by Alizadeh, they attributed that the decrease in FA may indicate degeneration of the fiber architecture, specifically the myelin will permit diffusion perpendicular to the neuronal axis, thus lowering the degree of FA. Although not statistically significant, the increase in MD may be attributed to an increase in cord edema [16].

Also, Facon previously found in their cohort no significant change in ADC values with a statistically significant difference for FA in patients with spinal cord injury and compression [17]. Werring and Beaulieu explained these findings in degenerated sciatic frog nerve and in human brain, a loss of anisotropy was noted, but average diffusivity may remain unchanged owing to an accumulation of cellular debris from a breakdown of the longitudinal axonal structure, while glial proliferation may hinder water movement in a parallel direction $[18,19]$. In vitro study was carried out on

\section{(See figure on next page.)}

Fig. 2 A 22-year-old male patient presented with low back pain of two-month duration, with progressive left foot drop. Sagittal and axial T2-weighted (A, C), sagittal T1-weighted image precontrast (B), sagittal, coronal axial, and post-contrast T1-weighted images (D-F). The terminal part of the spinal cord and conus medullaris region show expansile solid appearing space occupying mass lesion. The lesion shows bright T2 signal intensity with isointense signal intensity in T1WI. It shows irregular peripheral post-contrast enhancement. Diagnosis: Myxopapillary ependymoma. Sagittal trace $(\mathbf{G})$, ADC $(\mathbf{H})$, grayscale FA $(\mathbf{J})$, color-coded FA $(\mathbf{K})$ and 3D MR tractography $(\mathbf{L}, \mathbf{M})$ images. Trace image shows areas of mixed restricted and facilitated diffusion within the space occupying mass lesion. High ADC value and low FA values. The tractograms show widely separated fiber tracts with frank fiber disruption 


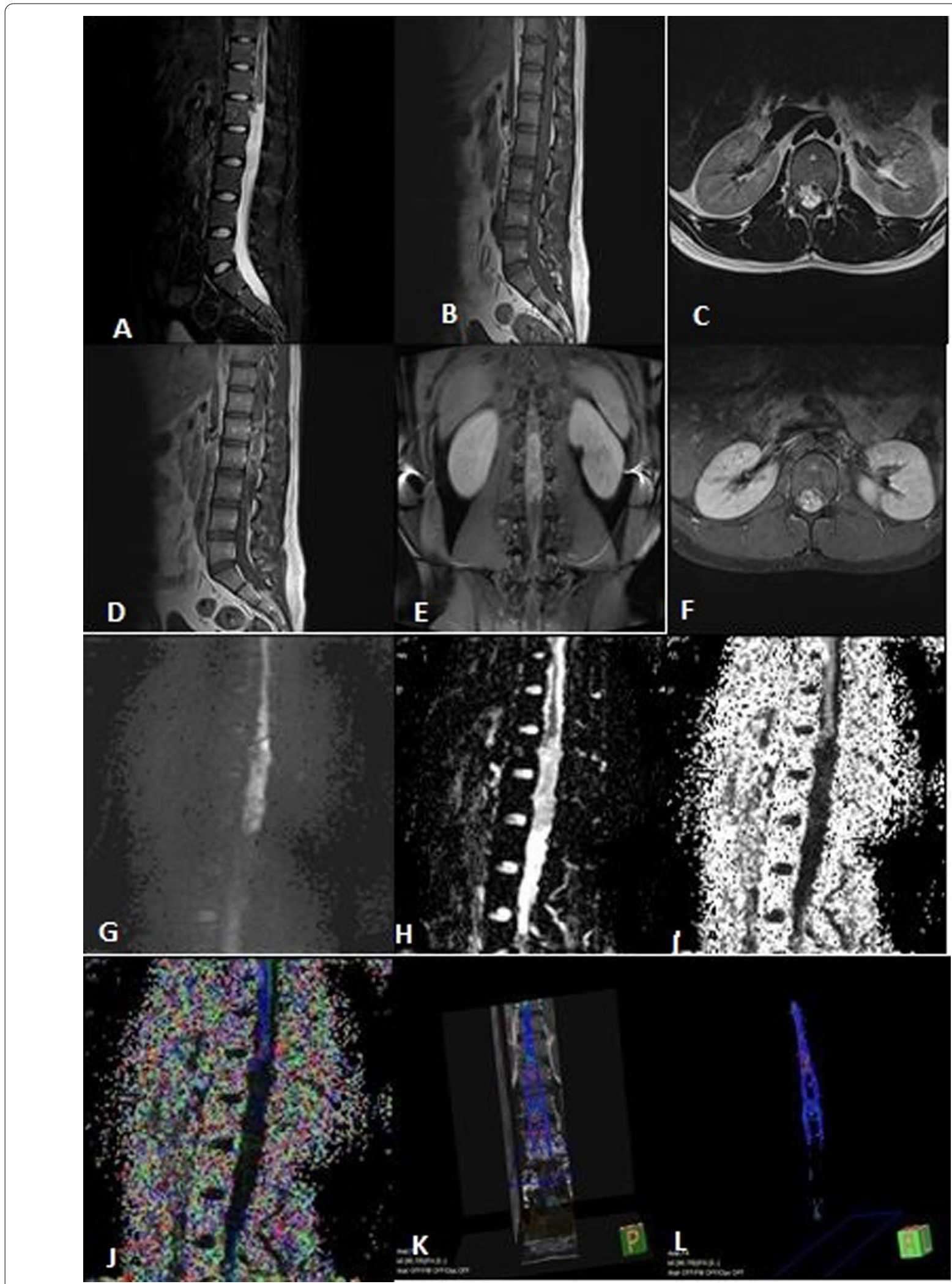

Fig. 2 (See legend on previous page.) 


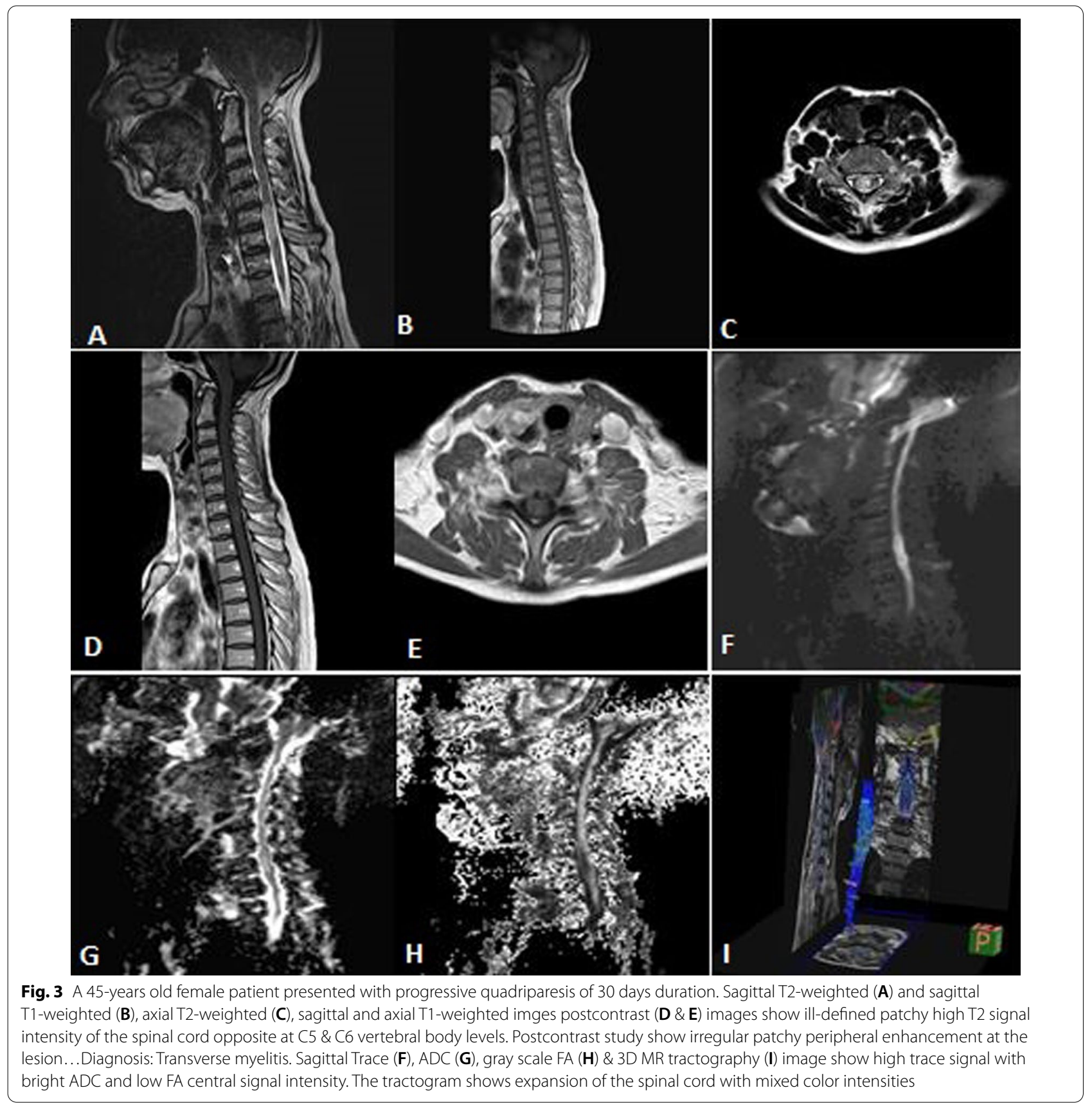

spinal cord specimens of Indian calf (Bos primigenius indicus). The specimens consisted of the spinal cord surrounded by the vertebral column and paraspinal muscles. In the compression injury sites, FA, RA and VR were significantly altered, and more importantly, there was a progressive decrease in the values as the severity of compression increased. On the contrary, the ADC and E1 values again did not show significant changes at the sites of pathology [20].
This agreed with our findings in each group of pathology studied separately, as Shanmuganathan findings with the FA and RA showing statistical significant differences in spinal cord injury with the greatest reduction were found at the site of spinal cord injury. Also they found significantly increased VR in the hemorrhagic injury site compared to those in control subjects, denoting an increased isotropic behavior at these sites. A high VR means isotropic diffusion. An increase in VR at the site of injury is consistent with corresponding 
Table 1 Repeated-measures ANOVA test comparing the difference in the calculated mean of different DTI metrics within the same group of pathology and between different pathology along the study cohort

\begin{tabular}{|c|c|c|c|}
\hline Parameter & $\begin{array}{l}\text { Trauma } \\
(n=12)\end{array}$ & $\begin{array}{l}\text { NTND } \\
(n=19)\end{array}$ & $p$ value* \\
\hline \multicolumn{4}{|l|}{ FA } \\
\hline At & $0.420 \pm 0.111$ & $0.471 \pm 0.115$ & $=0.150$ \\
\hline Above & $0.626 \pm 0.059$ & $0.587 \pm 0.095$ & $=0.272$ \\
\hline Below & $0.528 \pm 0.097$ & $0.522 \pm 0.143$ & $=0.859$ \\
\hline$p$ value $e^{* *}$ & $<0.001$ & $=0.007$ & \\
\hline \multicolumn{4}{|l|}{ ADC } \\
\hline At & $1.176 \pm 0.245$ & $1.161 \pm 0.121$ & $=0.978$ \\
\hline Above & $0.980 \pm 0.147$ & $1.078 \pm 0.109$ & $=0.080$ \\
\hline Below & $1.058 \pm 0.137$ & $1.137 \pm 0.164$ & $=0.477$ \\
\hline$p$ value $e^{* *}$ & $=0.318$ & $=0.220$ & \\
\hline \multicolumn{4}{|l|}{ RA } \\
\hline At & $0.375 \pm 0.108$ & $0.434 \pm 0.127$ & $=0.167$ \\
\hline Above & $0.601 \pm 0.132$ & $0.563 \pm 0.139$ & $=0.424$ \\
\hline Below & $0.491 \pm 0.119$ & $0.490 \pm 0.187$ & $=0.971$ \\
\hline$p$ value $e^{* *}$ & $<0.001$ & $=0.012$ & \\
\hline \multicolumn{4}{|l|}{ VR } \\
\hline At & $0.800 \pm 0.086$ & $0.740 \pm 0.116$ & $=0.213$ \\
\hline Above & $0.581 \pm 0.104$ & $0.617 \pm 0.104$ & $=0.431$ \\
\hline Below & $0.673 \pm 0.132$ & $0.694 \pm 0.164$ & $=0.918$ \\
\hline$p$ value $e^{* *}$ & $<0.001$ & $=0.034$ & \\
\hline
\end{tabular}

Repeated-measure ANOVA was used to compare the mean differences over time. The $A D C$ value $\times 10^{-3} \mathrm{~mm}^{2} / \mathrm{s}$

*Mean differences between group comparison

**Mean differences within group comparison

decreases seen in FA and RA when compared with control subjects. The RA followed FA trends and E1 mirror ADC value [10].

Loss of myelin integrity, which is typically secondary to demyelinating lesions, can be assessed with DTI. Demyelinating lesions demonstrate reduced FA values owing to a loss of anisotropy, as well as increased RD values secondary to an increase in water movement in the short axis, which occurs because the normal myelin barrier is damaged. On the contrary, after successful treatment, a decrease in $\mathrm{RD}$ and an increase in FA values occur with remyelination, indicating a positive response to treatment [21].

by Kang's result, which was previously described, was in agreement with our results. They observed significant increase in minor eigenvalues E2 and E3, signifying the increase in transverse diffusion and loss of anisotropism. The preservation of E1, or longitudinal diffusion, reflects the maintenance of axonal integrity [22]. Klawiter described a linear trend for increased $\lambda_{\perp}$ with increased demyelination and axonal loss within MS spinal cords
Table 2 Repeated-measures ANOVA test comparing the difference in the calculated mean of different DTI metrics within the same group of pathology and between different pathology groups along the study cohort

\begin{tabular}{llll}
\hline Parameter & Trauma $(\mathbf{n}=\mathbf{1 2})$ & NTND $(\mathbf{n}=\mathbf{1 9})$ & $\boldsymbol{p}$ value* \\
\hline E1 & & & \\
$\quad$ At & $1.721 \pm 0.288$ & $1.826 \pm 0.255$ & $=0.416$ \\
$\quad$ Above & $1.782 \pm 0.322$ & $1.889 \pm 0.214$ & $=0.355$ \\
$\quad$ Below & $1.732 \pm 0.241$ & $1.887 \pm 0.300$ & $=0.306$ \\
$\quad p$ value** & $=0.444$ & $=0.449$ & \\
E2 & & & \\
At & $1.048 \pm 0.283$ & $0.972 \pm 0.105$ & $=0.596$ \\
$\quad$ Above & $0.703 \pm 0.126$ & $0.807 \pm 0.113$ & $=0.163$ \\
$\quad$ Below & $0.909 \pm 0.285$ & $0.885 \pm 0.219$ & $=0.946$ \\
$\quad p$ value** & $=0.035$ & $=0.006$ & \\
E3 & & & \\
At & $0.778 \pm 0.213$ & $0.700 \pm 0.165$ & $=0.582$ \\
Above & $0.454 \pm 0.126$ & $0.538 \pm 0.117$ & $=0.052$ \\
Below & $0.543 \pm 0.136$ & $0.606 \pm 0.181$ & $=0.391$ \\
$\quad p$ value** & $=0.016$ & $=0.047$ & \\
\hline
\end{tabular}

RM ANOVA was used to compare the mean differences over time. The $\mathrm{E}$ values $\times 10^{-3} \mathrm{~mm}^{2} / \mathrm{s}$

*Mean differences between group comparison

**Mean differences within group comparison

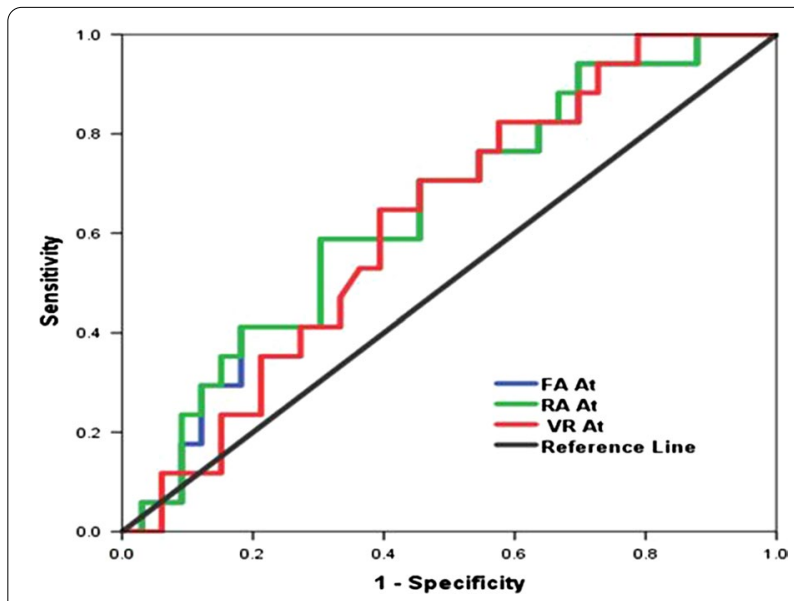

Fig. 4 ROC curve for DTI parameters at lesion versus conventional MRIT2WI detected myelopathy

[23]. In their study on neuromyelitis optica patient, Qian showed the reduced FA and increased increase in $\lambda_{\perp}$ (diffusivity perpendicular to the axonal fibers) in their cohort. This suggests that the most relevant pathological mechanism occurring in the spinal tracts of these patients was probably demyelination [24], while Naismith in their study showed highly increased radial diffusivity, 
Table 3 Diagnostic performance of DTI indices at lesion level versus $\mathrm{T} 2 \mathrm{WI}$ detected myelopathy, analyzed as area under the curve $(95 \% \mathrm{Cl})$

\begin{tabular}{lllll}
\hline & AUC & $95 \%$ Cl & SE & $p$ value \\
\hline FA & 0.642 & $0.512-0.801$ & 0.081 & $=0.041$ \\
ADC & 0.512 & $0.351-0.674$ & 0.083 & $=0.468$ \\
RA & 0.647 & $0.521-0.826$ & 0.081 & $=0.037$ \\
VR & 0.640 & $0.519-0.892$ & 0.081 & $=0.047$ \\
E1 & 0.564 & $0.404-0.742$ & 0.082 & $=0.261$ \\
E2 & 0.447 & $0.282-0.613$ & 0.085 & $=0.546$ \\
E3 & 0.479 & $0.316-0.641$ & 0.083 & $=0.406$ \\
\hline
\end{tabular}

AUC, area under the curve; SE standard error, $\mathrm{Cl}$ confidence interval

*Null hypothesis: true area $=0.5$

which has been associated with severe demyelination and axonal loss, correlating with the development of persistent T1 hypointensities or "black holes" in gadolinium enhancing brain lesions [25].

Klawiter described that, where $\lambda_{\|}$and $\lambda_{\perp}$ may be distended in a similar quantity, anisotropy may continue comparatively unchanged and so less informative. This is in distinction to other conditions where a differential increase in $\lambda_{\perp}$ or decrease in $\lambda_{\|}$may result in reduced anisotropy [23]. As Song found in their animal studies, we have reported that demyelination causes an increase in $\lambda_{\perp}$ without changing $\lambda_{\|}$[26]. Wang reported in their DTI animal study, which is in combination with biochemical validation, suggesting that increased $\lambda_{\perp}$ with no change in $\lambda_{\|}$represents an early sign of non-cystic white matter injury with reduced myelination before severe damage in structural integrity and necrosis [27].

When comparing the quantitative DTI indices between the two study groups of pathology, the results showed no statistically significant difference in the measured quantitative parameters for all indices. In agreeing with El Maati who found that the ADC and FA means of the tumor group were not significantly different from those of the non-tumor subjects $(p=0.539)$ [28]. In their study, Facon and Hassan showed decrease in FA value with variable $\mathrm{ADC}$ according to the type of extra-medullary tumor compressing the spinal cord [17, 29]. In agreeing with Facon, the FA measurements proved to have high sensitivity and specificity in the detection of spinal cord pathology in patients with extra-medullary spinal canal tumors [17].

In our findings, we support that the value of RA follows the trend of FA value and inverse to VR, while E1 mirrors ADC. The main contributing mechanism for FA reduction is the raised transverse diffusivity along the minor eigenvectors, which indicates the relative anisotropy and lowers the anisotropism. The preservation of E1, or longitudinal diffusion, reflects the maintenance of axonal integrity.

\section{Conclusions}

This study showed that the value of the newly additional sequence with its various measurable values ADC, FA, VR, RA and eigenvector values helps in depicting and supporting microstructure disorganization of various spinal cord lesions. We recommend DTI, whenever there is a focal abnormal signal in the cord, particularly when it shows post-contrast mass like enhancement. This study demonstrates the sensitivity of diffusivity along the minor axes (E2, E3 or $\lambda_{\perp}$ ) for the degree of demyelination, and injury of the spinal cord in contrast to (E1 or $\left.\lambda_{\| \mid}\right)$ did not show significant correlation supporting the loss of anisotropism with reduced FA and RA values. More follow-up of the same disease categorization of spinal cord lesions with these numerical DTI values was to provide applicable measures for disease prognosis.

\section{Abbreviations}

DTI: Diffusion tensor imaging; NTND: Non-traumatic-non-degenerative disease; FA: Fractional anisotropy; ADC: Apparent diffusion coefficient; VR: Volume ratio; RA: Relative anisotropy; E1: Longest eigenvector value; E2: Middle eigenvector value; E3: Minor eigenvector value; MRI: Magnetic resonance imaging; Wl: Weighted image; TR: Repetition time; TE: Time to echo; FOV: Field of view; TA: Acquisition time; 3D: Three-dimensional; ROI: Region of interest; SPSS: Statistical Program for Social Science; ROC: Receiver operator characteristic curve; ANOVA: Analysis of variance; SD: Standard deviation; MD: Mean diffusivity; RD: Radial diffusivity; $\lambda \Perp$ : Diffusion perpendicular to axonal fiber axis; $\lambda_{\|}$: Diffusion parallel to axonal fiber axis; MS: Multiple sclerosis; AUC: Area under the curve; $\mathrm{Cl}$ : Confidence interval; SE: Standard error; n: Number.

\section{Acknowledgements}

Not applicable.

\section{Authors' contributions}

AKH was the major contributor in writing the manuscript and providing structural advices. MZ helped in data analysis and interpretation of the study. MK helped in body research construction and guideline. All authors have read and approved the manuscript, ensure that this is the case. All authors read and approved the final manuscript.

\section{Funding}

Not applicable.

\section{Availability of data and materials}

The data that support the findings of this study are available and not for public view.

\section{Declarations}

Ethics approval and consent to participate

The study protocol was approved by the Research Ethics Board of faculty of medicine-Assiut University (IRB number 17200249), assuring respect of the confidentiality of the medical record, and informed written consent was obtained from every participant.

Consent for publication

All patients included in this research gave written informed consent to publish the data contained within this study. 
Competing interests.

The authors declare that they have no competing interests.

\section{Author details}

${ }^{1}$ Assiut University, Asyût, Egypt. ${ }^{2}$ Luxor University, Luxor, Egypt.

Received: 24 May 2021 Accepted: 21 August 2021

Published online: 12 November 2021

\section{References}

1. Seidenwurm DJ (2008) Myelopathy. AJNR Am J Neuroradiol 29(5):1032-1034

2. Jones DK (2010) Diffusion mri. Oxford University Press

3. Zhang C et al (2014) Application of magnetic resonance imaging in cervical spondylotic myelopathy. World J Radiol 6(10):826-832

4. Zhao $\mathrm{M}$ et al (2017) Axial MR diffusion tensor imaging and tractography in clinical diagnosed and pathology confirmed cervical spinal cord astrocytoma. J Neurol Sci 375:43-51

5. Zheng W et al (2019) Diffusion tensor magnetic resonance imaging in chronic spinal cord compression. J Vis Exp (147)

6. Banaszek A et al (2016) Role of diffusion tensor MR imaging in degenerative cervical spine disease: a review of the literature. Clin Neuroradiol 26(3):265-276

7. Rindler RS et al (2017) Spinal diffusion tensor imaging in evaluation of preoperative and postoperative severity of cervical spondylotic myelopathy: systematic review of literature. World Neurosurg 99:150-158

8. Sasiadek MJ, Szewczyk P, Bladowska J (2012) Application of diffusion tensor imaging (DTI) in pathological changes of the spinal cord. Med Sci Monit 18(6):73-Ra73-9

9. D'Souza MM et al (2017) Diffusion tensor MR imaging in spinal cord injury. Injury 48(4):880-884

10. Shanmuganathan $\mathrm{K}$ et al (2008) Diffusion tensor MR imaging in cervical spine trauma. AJNR Am J Neuroradiol 29(4):655-659

11. Rajasekaran $S$ et al (2014) The assessment of neuronal status in normal and cervical spondylotic myelopathy using diffusion tensor imaging. Spine (Phila Pa 1976) 39(15):1183-1189

12. Ohgiya $Y$ et al (2007) Diffusion tensor MR imaging of the cervical spinal cord in patients with multiple sclerosis. Eur Radiol 17(10):2499-2504

13. Rutman AM et al (2018) Diffusion Tensor Imaging of the Spinal Cord: Clinical Value, Investigational Applications, and Technical Limitations. Curr Probl Diagn Radiol 47(4):257-269

14. Ducreux D et al (2007) Diffusion tensor magnetic resonance imaging and fiber tracking in spinal cord lesions: current and future indications. Neuroimaging Clin N Am 17(1):137-147
15. Setzer M et al (2010) Diffusion tensor imaging tractography in patients with intramedullary tumors: comparison with intraoperative findings and value for prediction of tumor resectability: Presented at the 2009 Joint Spine Section Meeting. J Neurosurg Spine 13(3):371-380

16. Alizadeh $\mathrm{M}$ et al (2015) Intensity inhomogeneity correction in clinical pediatric spinal cord MRI images. In: 2015 41st annual northeast biomedical engineering conference (NEBEC). IEEE

17. Facon $D$ et al (2005) MR diffusion tensor imaging and fiber tracking in spinal cord compression. Am J Neuroradiol 26(6):1587-1594

18. Werring DJ et al (2000) Diffusion tensor imaging can detect and quantify corticospinal tract degeneration after stroke. J Neurol Neurosurg Psychiatry 69(2):269-272

19. Beaulieu C et al (1996) Changes in water diffusion due to Wallerian degeneration in peripheral nerve. Magn Reson Med 36(4):627-631

20. Rajasekaran S et al (2012) Efficacy of diffusion tensor anisotropy indices and tractography in assessing the extent of severity of spinal cord injury: an in vitro analytical study in calf spinal cords. Spine J 12(12):1147-1153

21. Noguerol TM et al (2020) Optimizing diffusion-tensor imaging acquisition for spinal cord assessment: physical basis and technical adjustments. Radiographics 40(2):403-427

22. Kang M, Anderer E, Elliott R, Kalhorn S, Cooper P, Frempong-Boadu A Diffusion tensor imaging of the spondylotic cervical spinal cord: a preliminary study of quantifiable markers in the evaluation for surgical decompression. Internet J Head Neck Surg 5(1)

23. Klawiter EC et al (2011) Radial diffusivity predicts demyelination in ex vivo multiple sclerosis spinal cords. Neuroimage 55(4):1454-1460

24. Qian W et al (2011) Quantitative assessment of the cervical spinal cord damage in neuromyelitis optica using diffusion tensor imaging at 3 Tesla. J Magn Reson Imaging 33(6):1312-1320

25. Naismith $\mathrm{R}$ et al (2010) Increased diffusivity in acute multiple sclerosis lesions predicts risk of black hole. Neurology 74(21):1694-1701

26. Song S-K et al (2005) Demyelination increases radial diffusivity in corpus callosum of mouse brain. Neuroimage 26(1):132-140

27. Wang S et al (2008) Characterization of white matter injury in a hypoxic-ischemic neonatal rat model by diffusion tensor MRI. Stroke 39(8):2348-2353

28. El Maati AAA, Chalabi N (2014) Diffusion tensor tractography as a supplementary tool to conventional MRI for evaluating patients with myelopathy. Egypt J Radiol Nucl Med 45(4):1223-1231

29. Hassan HG et al (2020) The role of diffusion tensor imaging (DTI) in spinal cord pathology. Ain Shams Med J 71(1):197-205

\section{Publisher's Note}

Springer Nature remains neutral with regard to jurisdictional claims in published maps and institutional affiliations.

\section{Submit your manuscript to a SpringerOpen ${ }^{\circ}$ journal and benefit from:}

- Convenient online submission

- Rigorous peer review

- Open access: articles freely available online

- High visibility within the field

Retaining the copyright to your article

Submit your next manuscript at springeropen.com 